\title{
A Novel Method to Adjust Saphenous Vein Graft Lengths Using 3D Printing Models
}

\author{
Hakan Gocer, MD,${ }^{1}$ Ahmet Baris Durukan, MD, Associate Professor, ${ }^{2}$ Osman Tunc, ${ }^{3}$ Erdinc Naseri, MD, ${ }^{4}$ \\ Ertugrul Ercan, MD, Professor ${ }^{5}$ \\ ${ }^{1}$ Medical Park Usak Hospital, Department of Cardiology, Usak, Turkey; ${ }^{2}$ Medical Park Usak Hospital, Department of Cardiovascular \\ Surgery, Usak, Turkey; ${ }^{3}$ BTECH Company, Middle East Technical University, Techno City, Ankara, Turkey; ${ }^{4}$ Afyon Park Hospital, \\ Department of Cardiovascular Surgery, Afyon, Turkey; ${ }^{5}$ Medical Park İzmir Hospital, Department of Cardiology, İzmir, Turkey
}

\section{ABSTRACT}

Background: The optimal length of saphenous vein grafts can be challenging in surgical coronary revascularization. It is the cornerstone for graft patency. In this study, we tried to demonstrate the value of $3 \mathrm{D}$ printing in determining optimal saphenous graft length.

Methods: Sixteen patients who underwent bypass surgery with only vein grafts were examined. Patients' measurements of graft lengths were obtained from postoperative CT images and from both $3 \mathrm{D}$ print models manually with plastic tubes and via $3 \mathrm{D}$ print digital images of Mimics software during segmentation. Another measurement was done using the Fit Centerline tool in the analysis module of Mimics software after segmentation. These 3 measurements were compared.

Results: There was a statistically significant difference between 3 measurement methods for each graft length $(P<.001)$. Measurements of actual grafts were longer than measurements of $3 \mathrm{D}$ printed models manually and segmentation images from software were similar $(P>.05)$.

Conclusion: 3D printing models and their software may be used to determine optimal saphenous graft length and the anastomosis site to decrease operation time. It can be deducted from these results that $3 \mathrm{D}$ printing is a promising method for reducing operator dependent variables in adjusting graft size and finding optimal anastomosis sites.

\section{INTRODUCTION}

Patients with multiple native coronary artery lesions need multiple grafts, during coronary artery bypass grafting (CABG) [El Bardies 2012]. Multiple arterial grafts are used in approximately $5 \%$ to $10 \%$ of patients undergoing CABG. Yet, saphenous vein graft (SVG) still is the preferred main conduit [El Bardies 2012; Hess 2014]. It usually is available at the desired length that is roughly measured and adjusted by the surgeon during operation [Bourassa 1985]. Saphenous

Received October 10, 2019; accepted fanuary 4, 2020.

Correspondence: Abmet Baris Durukan, Umit Mahallesi, 2463. sokak 4/18, 06520, Cankaya, Ankara/Turkey; +905322273814 (e-mail: barisdurukan@ yahoo.com). graft failure may be as high as $25 \%$ in the first 12 to 18 months. Late failure also is a concern and can reach up to 50\% [Lopes 2012; Halabi 2005]. It also was documented that SVG failure is correlated with unfavorable outcomes [Halabi 2005; Harskamp 2013]. The main cause of acute SVG failure is acute graft thrombosis, during the first month. Focal neointimal hyperplasia may cause failure at the anastomotic sites in between 1 month and 2 years. Atherosclerotic degeneration with neointimal hyperplasia is the main reason after 2 years [Harskamp 2013; Sabik 2011].

Accurate SVG length adjustment is crucial, since both a too short and a too long graft affect patency unfavorably [Goor 2007; Milo 1982]. The slightest mismatch of SVG length can cause systolic kinking or slight tension that requires readjustment of length [Milo 1982; Bonchek 1978]. In a living human, surgeons use a combination of the coronary angiogram, visual inspection, and palpation to determine the ideal location of grafts and several techniques have been reported to overcome graft length misjudgment. One of these is the Goor's technique that is employed to optimize saphenous vein graft length [Milo 1982; Bonchek 1978]. Despite these techniques and operator experience, SVG length and anastomosis site location still depend on operator's subjective estimation.

Three-dimensional printing first was described by Charles Hull in 1986 [Vukicevic 2017]. It is a fabrication technique used to transform digital images into physical objects [Anwar 2018; Maragiannis 2015]. It consists of several steps, which begin with using the "Digital Imaging and Communication in Medicine" (DICOM) files from the volumetric images data of computed tomography (CT), magnetic resonance imaging (MRI), and ultrasound [Gosnell 2016; Firouzian 2016]. These data images are processed using segmentation and mesh generation with special software followed by conversion to a standard tessellation language file (STL) for printing [Sodian 2007; Greil 2007]. Stereo lithography, selective laser sintering, inkjet, and fuseddeposition modeling are different options of medical 3D printing technologies with different printers [Binder 2000; Byrne 2016].

The software packages approved by FDA for medical purposes and measurements used for segmentation and further digital processing are superior to different radio-diagnostic tool software in making real measurements [Vukicevic 2017; 


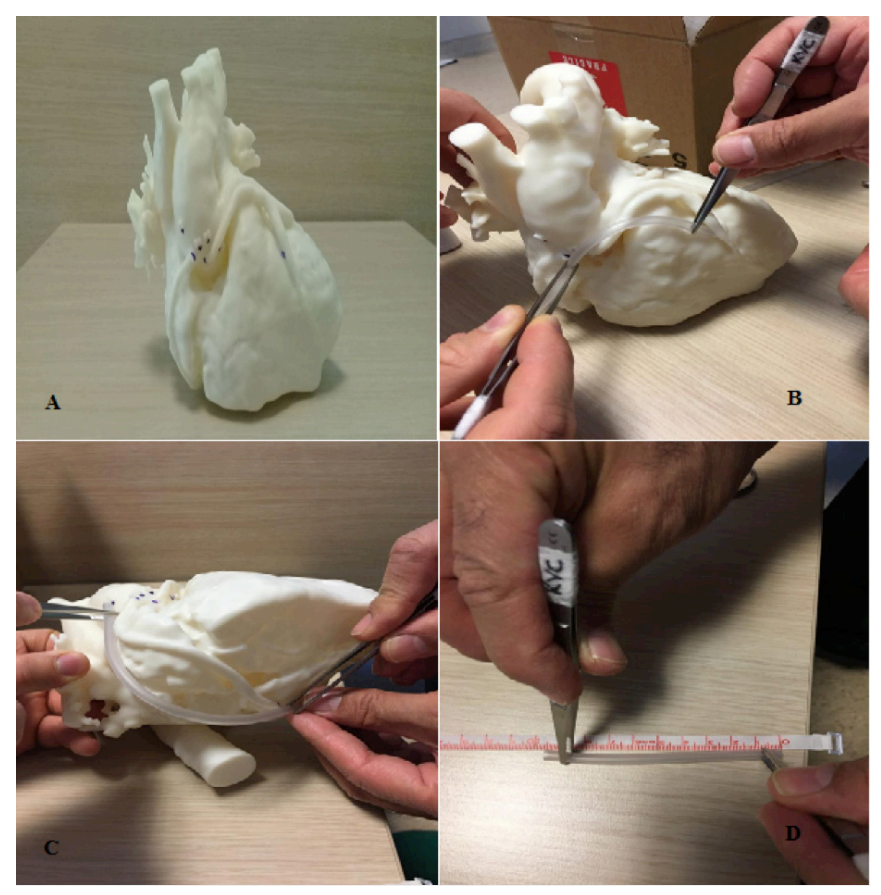

Figure 1. A, 3-D Printed model of heart, aorta and coronary arteries; $B$, Manual estimation of optimal location and length of Ao-saphenous graft-LAD/D1 via plastic tube; C, Manual estimation of optimal location and length of Ao-saphenous graft-RCA/PDA via plastic tube; D, Manual measurements of estimated optimal Ao-saphenous graft-LAD/ D1 length via ruler.

Gosnell 2016]. The software enables more accurate "surface to surface" virtual measurements without printing physical models. But there is no current study in this area on CABG surgery, especially in terms of virtual and manual estimation of graft structure, length and also planning of surgical technique [Vukicevic 2017; Byrne 2016].

Cardiovascular applications of $3 \mathrm{D}$ printing still are a relatively new area and can be used for complex procedural planning in congenital or acquired cardiovascular disease, creating and refining of anatomic teaching tools and intra-cardiac devices, developing functional models to investigate intracardiac flows and non-sterile practicing before operations in patient-specific models [Goor 2007; Milo 1982; Bonchek 1978; Vukicevic 2017; Anwar 2018; Maragiannis 2015; Gosnell 2016; Firouzian 2016; Sodian 2007; Greil 2007; Binder 2000; Byrne 2016; Hadeed 2018].

We hypothesized that a preoperative 3D print model of the heart, native coronary vessels, and aortic root can play an important role in adjusting graft length. To show the accuracy of this hypothesis, we evaluated postoperative CT images of 16 patients who underwent CABG with multiple SVGs, retrospectively. We measured optimal graft length on $3 \mathrm{D}$ print models both manually and on digital images via Materialise software and compared these measurements and calculations with actual patients' postoperative CT graft lengths and anastomosis sites.

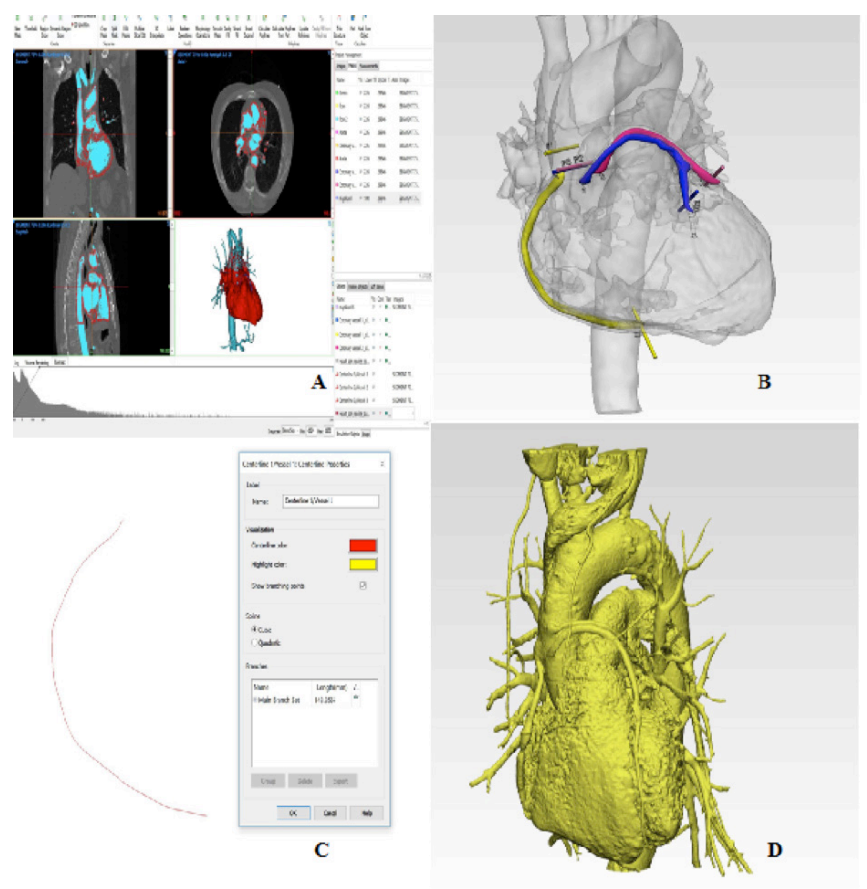

Figure 2. A, Segmentation of heart, myocardium, coronary arteries and saphenous grafts (region of interest) via Mimics software; B, Segmentation of saphenous grafts and marking optimal anastomosis sites via Mimics software; C, Centerline command of Mimics software in measuring graft length; D, Volumetric images of coronary arteries and grafts via computerized tomography angiography.

\section{METHODS}

This retrospective study was conducted in Izmir Medical Park Hospital and Middle East Technical University, using BTECH 3D printing company in November 2018. The study was approved by the ethical committee of the hospital waiving informed consent due to retrospective nature of the study.

Sixteen patients who previously had undergone CABG in the same institution by the same surgeon were included. Patients who only had SVGs particularly were selected in this retrospective study because the aim to evaluate SVGs. In these patients, the left internal mammary artery was not used for different reasons, including severe terminal aortic disease, severe chronic obstructive pulmonary disease, left subclavian artery stenosis, and poor flow. Excluded were redo cases, patients who had thoracic surgery, and those with thoracic deformity. All patients had 4 SVGs and were operated on pump. Saphenous graft distal anastomosis sites were left anterior descending (LAD), circumflex posterolateral branch, first diagonal artery, and right posterior descending artery. The proximal anastomosis site was ascending aorta for all patients. Contrast CT images were obtained for different reasons within 30 days following surgery. A Toshiba Aquiline One Vision Edition 640 slice Dynamic volume tomography was used for contrast CT images of the heart, native vessels, myocardium, aorta, and saphenous grafts. Mimics Innovation Suite 21 software (Materialise, Leuven, Belgium, 
Table 1. Comparison of SVG length measurements with three methods

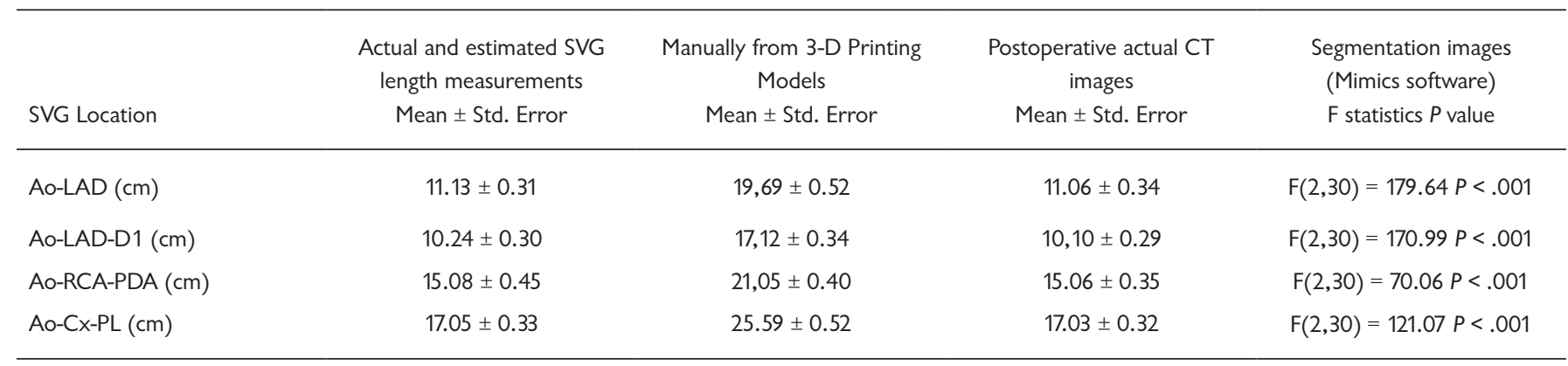

SVG: saphenous vein graft, CT: computerized tomography, LAD: left anterior descending artery, D1: first diagonal artery, RCA-PDA: posterior descending artery branch of right coronary artery, Cx-PL: posterolateral branch of circumflex coronary artery

and CE 0120 Certification) was used in this study, during segmentation design, modeling and making measurements.

General steps of the software and printing in this study: First, the DICOM files of diagnostic CTs were imported to Mimics software followed by the creation of threedimensional models of the aorta, myocardium, SVGs, coronary arteries, and anastomosis sites by the software. The length of the saphenous grafts in between the proximal and distal anastomosis sites was measured with software. The model created in Mimics was exported to the design module of 3-matic and the command of smooth, wrap, and fix was used to design. Last state of the design in final STL file format was exported to software (preform) of the resin based laser technology (SLA) 3D printer (Formlabs Form 2, Formlabs Inc. Massachusetts, USA, and Laser Specifications: EN 60825-1:2007certified, Class 1 laser product $405 \mathrm{~nm}$ violet laser, $250 \mathrm{~mW}$ laser). After orientation and support procedures, the production of the model was made from standard resin and white resin. Support points were then detached after washing and a curing process.

Using the aforementioned physical model and software, we evaluated graft length via 2 methods, where proximal and distal anastomoses sites were same as the actual. One method was manual measurement of the gap between the distal and proximal optimal anastomosis sites with plastic tubes having similar structure to SVGs like serum sets by 2 experienced cardiovascular surgeons, one of whom performed CABG (Figure 1). The second measurement method was performed by a mechanical engineer with 2 cardiac surgeons and 2 cardiologists, who are experienced in $3 \mathrm{D}$ printing and software on $3 \mathrm{D}$-constructed images of digital printing models with Materialise software (Figure 2). Data from both techniques and actual postoperative CT image measurements were compared.

Statistical analysis was performed using SPSS software for Windows version 17.0 (Statistical Package for the Social Sciences Inc, Chicago, IL, USA). All parametric results are expressed as the mean \pm standard error except for demographic characteristics given as Mean $\pm \mathrm{SD}$. Univariate analysis was performed using Two Way Variance Analysis (ANOVA).

\section{RESULTS}

The mean age of the patients was $64.5 \pm 2.15$. Their preoperative echocardiographic left ventricular ejection fraction and body mass index were $54.69 \pm 1.16 \%$ and $27.75 \pm 0.75 \mathrm{~kg} /$ $\mathrm{m}^{2}$ at admission, respectively.

When 3 measurement methods were compared, the actual SVG length measurements were longer than measurements made on the 3D print model and digital model for each graft $(P<.001$ for each) (Table 1$)$. In Table 2, each measurement method was compared for every graft. Actual SVG length was longer than $3 \mathrm{D}$ print model measurement $(P<.001)$ and software measurement $(P<.001)$ for each graft. When the measurements obtained from 3D printed models manually and measurements of segmentation images from Materialise software were compared, the measurements were similar for each graft $(P>.05)$.

\section{DISCUSSION}

$3 \mathrm{D}$ printing is a novel technology and widely is used in the industry. Medical applications have gained acceptance in oncology, orthopedics, cardiology, anatomy, and vascular surgery [Vukicevic 2017]. It is used for planning treatment strategy and operations, educational purposes, and performing interventions in non-sterile environments preoperatively [Maragiannis 2015; Mahmood 2015; Vukicevic 2017].

$3 \mathrm{D}$ printing technology continuously is developing, especially in the field of printer's software and framework, printing material structure, and processing software. Segmentation is the main step of 3D printing [Vukicevic 2017]. You can convert diagnostic DICOM files to printing files (STL) and measure and calculate actual distance, volumes, and mass during segmentation. Also, these measurements are superior to conventional imaging tools (CT, MRI, and 3D echocardiography) [Vukicevic 2017; Gosnell 2016; Binder 2000; Byrne 2016].

After segmentation, files are sent to the printer, and the printing process begins. Special laser printers commonly are used. We also employed this standard procedure as the method. 
Table 2. Comparison of SVG length with three measurement methods: Post-Hoc analysis

\begin{tabular}{|c|c|c|c|}
\hline CT & $P<.001$ & - & \\
\hline Software & NS & $P<.001$ & - \\
\hline Ao-LAD-D1 $(\mathrm{cm})$ & Manual & CT & Software \\
\hline Manual & - & & \\
\hline Software & NS & $P<.001$ & - \\
\hline Ao-RCA-PDA (cm) & Manual & CT & Software \\
\hline Manual & - & & \\
\hline $\mathrm{CT}$ & $P<.001$ & - & \\
\hline CT & $P<.001$ & - & \\
\hline Software & NS & $P<.001$ & - \\
\hline
\end{tabular}

SVG: saphenous vein graft, CT: computerized tomography, LAD: left anterior descending artery, D1: first diagonal artery, RCA, PDA: posterior descending artery branch of right coronary artery, Cx-PL: posterolateral branch of circumflex coronary artery, NS: non-significant

Standard resin and white resin are widely used materials for printing. They are cost effective and easy to form. When you have an actual 3D printed model, you can make a practice plan and more accurate measurements manually may be performed. This gains operator self-confidence and speed during real operation [Kappanayil 2017].

$3 \mathrm{D}$ printing frequently is used in the field of cardiology. However, cardiovascular surgeons have begun to pay more attention, especially in planning procedures, practicing medical instruments, and developing medical devices. Cardiovascular surgeons may be able to solve problems in complex cases by the advancements in printing materials (more soft elastomeric polymers) and printer technology (combine different structure with more realism). This gives the opportunity to make more realistic complex combined models [Kappanayil 2017]. Coronary segmentation and printing in coronary anomalies were done [Lee 2019], but native arteriosclerotic coronary segmentation and planning procedures on coronary arteries have not been performed. To the best of our knowledge, this is the first study to segment and print native coronary arteries for planning surgical revascularization. This gave us the opportunity to evaluate length of grafts via printed models and software. In our study, we tried to demonstrate the importance of predetermining SVG length. This issue still is debatable. Spatial geometry is variable during surgery, and graft length continues to be relevant for long-term graft patency. Surgeons still use their experience and knowledge, when finding the optimal anastomosis site and determining graft length. This results in dependency on the operator's skills and personal variability. Our results show that predetermination of graft length with modules of segmentation software before printing, and manually on 3D printed models after printing are valuable methods that may provide better long-term graft patency by preventing possible kinking and tension. Therefore, predetermination of optimal SVG length may decrease operation time, and assuming provision of better graft patency, may decrease morbidity and mortality. We used Mimics Innovation Suite 21 Software (Materialise, Leuven, Belgium, and CE 0120 Certification) for segmentation and measurements. This software is the only approved and certified software for medical designs and producing medical products from the FDA and CE. Also, we used 3D printer (Formlabs Form 2, Formlasbs Inc. Massachusetts, USA, and Laser Specifications: EN 60825-1:2007certified, Class 1 laser product $405 \mathrm{~nm}$ violet laser, $250 \mathrm{~mW}$ laser). This printer is FDA certified for production of medical products. Furthermore, our printing materials (standard resin and white resin) also are FDA certified.

This hypothesis must be evaluated with further prospective big studies. Also, engineering and medical collaboration must be provided. Some big hospitals recently have established 3D printing labs. These labs are cost effective, but need support from the medical and technological community. This technology may offer important additional perspective to develop technologies of cardiac imaging, image processing software, a combined dual-material $3 \mathrm{D}$ printing, and several patientspecific models [Vukicevic 2017]. Patient-specific 3D real-time moving models via virtual reality may directly impact our ability to select appropriate patients for structural heart therapy, anticipate procedural complications, and potentially revise and improve cardiac therapeutic devices in CABG surgery besides congenital structural heart disease [Binder 2000; Byrne 2016]. 
Limitations: Our study has some drawbacks. Due to the retrospective nature and small scale, our hypothesis like reducing mortality, morbidity, and operation time, graft patency must be tested further in prospective big studies. The models are not dynamic, so we solely evaluated end systolic dimensions. 3D printing technology still is developing in parallel to radiological imaging techniques that are sources for $3 \mathrm{D}$ printing. In the future, the printing process may be shortened and costs may be reduced. Moreover, left internal mammary artery grafts could be evaluated with more comprehensive models in large-scale studies. Our study only retrospectively tested SVGs. Each model cost about $300 \mathrm{~s}$ and took 6 hours to print in this study. The expenses and manufacturing time must be reduced with advanced and separate technology, so this particular technology can be used in real life.

\section{CONCLUSION}

The technology represented here may be effective in preoperative determination of SVG length. This may decrease operative time and increase graft patency.

\section{REFERENCES}

Anwar S, Singh GK, Miller J, Sharma M, Manning P et al. 2018. 3D Printing is a Transformative Technology in Congenital Heart Disease. JACC Basic Transl Sci 3:294-312.

Binder TM, Moertl D, Mundigler G, Rehak G, Franke M et al. 2000. Stereolithographic biomodeling to create tangible hard copies of cardiac structures from echocardiographic data: in vitro and in vivo validation. $\mathrm{J}$ Am Coll Cardiol 35:230-7.

Bonchek LI, Olinger GN. 1978. Technical considerations in saphenous vein bypass grafts to the right coronary artery. Ann Thorac Surg 25:254-6.

Bourassa MG, Fisher LD, Campeau L, Gillespie MJ, McConney M et al. 1985. Long-term fate of bypass grafts: the Coronary Artery Surgery Study (CASS) and Montreal Heart Institute experiences. Circulation 72 V71-8.

Byrne N, Velasco FM, Tandon A, Valverde I, Hussain T. 2016. A systematic review of image segmentation methodology, used in the additive manufacture of patient-specific 3D printed models of the cardiovascular system. JRSM Cardiovasc Dis 5:204800401664.

El Bardies AW, Armani SF, Sheng S, O'Brien SM, Greenberg CC et al. 2012. Trends in isolated coronary artery bypass grafting: an analysis of the Society of Thoracic Surgeons adult cardiac surgery database. J Thorax Cardiovascular Surg. 143 273-81.

Firouzian A, Manniesging R, Flach ZH, Risselada R, Kooten F. 2016. Intracranial aneurysm segmentation in 3D CT angiography: method and quantitative validation with and without prior noise filtering. Eur J Radiol 79:299-304.

Goor DA. 2007. The importance of the vein graft torsion (addendum to chapter XVIII). in: D.A. Goor (Ed.)The Genius of C. Walton Lillehei and the True History of Open Heart Surgery. 1st ed. Vantage Press, New York 295-297.
Gosnell J, Pietila T, Samuel BP, Kurup HK, Haw MP et al. 2016. Integration of Computed Tomography and Three-Dimensional Echocardiography for Hybrid Three-Dimensional Printing in Congenital Heart Disease. J Digit Imaging 29:665-9.

Greil GF, Wolf I, Kuettner A, Fenchel M, Miller S et al. 2007. Stereolithographic reproduction of complex cardiac morphology based on high spatial resolution imaging. Clin Res Cardiol. 96:176-85.

Hadeed K, Acar P, Dulac Y, Cuttone F, Alacoque X et al. 2018. Cardiac $3 \mathrm{D}$ printing for better understanding of congenital heart disease. Arch Cardiovasc Dis 111:1-4.

Halabi AR, Alexander JH, Shaw LK, Lorenz TJ, Liao L et al. 2005. Relation of early saphenous vein graft failure to outcomes following coronary artery bypass surgery. Am J Cardiol. 96: 1254-9.

Harskamp RE, Lopes RD, Baisden CE, de Winter RJ, Alexander JH. 2013. Saphenous vein graft failure after coronary artery bypass surgery: pathophysiology, management, and future directions. Ann Surg 257:824-33.

Hess CN, Lopes RD, Gibson CM, Hager R, Wojdyla DM et al. 2014. Saphenous vein graft failure after coronary artery bypass surgery: insights from PREVENT IV. Circulation 130:1445-51.

Kappanayil M, Koneti NR, Kannan RR, Kottayil BP, Kumar K. 2017. Three-dimensional-printed cardiac prototypes aid surgical decisionmaking and preoperative planning in selected cases of complex congenital heart diseases: Early experience and proof of concept in a resourcelimited environment. Ann Pediatr Cardiol 10:117-25.

Lee M, Moharem-Elgamal S, Beckingham R, Hamilton M, Manghat $\mathrm{N}$ et al. 2019. Evaluating 3D-printed models of coronary anomalies: a survey among clinicians and researchers at a university hospital in the UK. BMJ Open 9:e025227.

Lopes RD, Mehta RH, Hafley GE, Williams JB, Mack MJ et al. 2012. Relationship between vein graft failure and subsequent clinical outcomes after coronary artery bypass surgery. Project of Ex Vivo Vein Graft Engineering via Transfection IV (PREVENT IV) Investigators. Circulation 125:749-56.

Mahmood F, Owais K, Taylor C, Montealegre-Gallegos M, Manning W et al. 2015. Three-dimensional printing of mitral valve using echocardiographic data. JACC Cardiovasc Imaging 8:227-9.

Maragiannis D, Jackson MS, Igo SR, Schutt RC, Connell P et al. 2015. Replicating Patient-Specific Severe Aortic Valve Stenosis With Functional 3D Modeling. Circ Cardiovasc Imaging. 8:e003626.

Milo S, Massini C, Goor DA. 1982. Coronary vein graft marking: a method to prevent graft twisting and length misjudgment. Ann Thorac Surg 33:200-2.

Sabik JF III. 2011. Understanding saphenous vein graft patency. Circulation 124:273-5.

Sodian R, Weber S, Markert M, Rassoulian D, Kaczmarek I et al. 2007. Stereolithographic models for surgical planning in congenital heart surgery. Ann Thorac Surg 83:1854-7.

Vukicevic M, Bobak Mosadegh B, James K, Little SH. 2017 Cardiac 3D Printing and Its Future Directions. JACC Cardiovasc Imaging 10:171-84.

Vukicevic M, Puperi DS, Jane Grande-Allen K, Little SH. 2017. 3D Printed Modeling of the Mitral Valve for Catheter-Based Structural Interventions. Ann Biomed Eng 45:508-19. 